more cases. Out of this total of twelve cases done in the last 4 to 5 months-eight were intracapsular extractions, and four extracapsular. In one of the intracapsular cases there was some vitreous loss after the lens was extracted, but in neither this case nor any of the others were there any unusual symptoms, and the end results were excellent.

In the last eleven cases before the suture was finally drawn tight, gtt. penicillin and gtt. atropine were instilled into the mouth of "the bag." A small point which led to a little trouble with fixation of the eye in the first case, was that a small tag of conjunctiva should be left on the corneal margin at the point the operator usually prefers to place his fixation forceps-otherwise he finds it difficult to grip the eye without doing so on one of the recti muscles.

The suture was usually found to give about the 4th or 5th day, and the conjunctiva had retracted to the corneal margin by the 6th or 7th day. In only one case-for ultra-aesthetic reasons-was it necessary to help the conjunctiva back into place at one point on the 10 th day.

The method is admittedly more tedious than the usual ones in vogue, but the feeling of complete security is very real. The purse string suture is easy to draw tightly and snugly, and the pressure over the whole cornea and wound is even and firm. The physiological conditions for wound healing are therefore ideal inasmuch as it is covered, supported, warm and sheltered from infection.

The fact that the patients can be ambulatory with safety after the first twenty-four hours is a marked advantage in these days of depleted nursing staffs, and any post-operative complications which may arise-with the exception of infection-are in eftect dealt with before they happen.

I very much doubt if this procedure is original since nearly everything has been tried sometime, but I have not seen any reference to it in the literature available to me, and it is presented in the hope that it may be found to be a useful addition to the usual methods.

\title{
THE POWER OR "POWERS" OF" A LENS
}

BY

Joseph I. Pascal, M.D.

NEW YORK

IF a doctor at an Ophthalmological Board examination were asked, "What is the power of the crystalline lens?", he might well ask which of the different "powers" of the lens was wanted. In situ the lens may be said to have three powers, based on actual focal 
lengths, i.e. the principal power, the vertex power, and the apex power. Placed in air it is very much more powerful, and again may be said to have three powers, the principal power, the vertex power, and the apex power. But in addition, and this is the most important point, in situ the lens has also a "reduced" power. This is the power as determined by the reduced focal length. This latter power is the real significant power and the one used by Gullstrand in his calculations of the dioptric power of the eye.

The definition of surface power and lens power, following the lead of Gullstrand, is based upon " reduced focal length". The power in dioptres is the reciprocal of the reduced focal length. The two actual focal lengths of any optical system are equal, when the first medium (or its index of refraction) is the same as the last medium (or same index of refraction). The two focal lengths are unequal when the first and last media are different. But in either case, there is only one reduced focal length. The refractive power of the system can be expressed by a single value, the reciprocal of this focal length, even when there are two actual focal lengths as in the case of the cornea, or the eye as a whole.

The reduced focal length is simply defined as the actual focal length divided by the corresponding index of refraction. Thus if $f_{1}$ and $f_{2}$ express the two actual focal lengths of a system, the reduced focal length $f(r)$ is equal to $f_{1}$ divided by $n_{1}$ or $f_{2}$ divided by $n_{2}$. The whole subject of "reduced" distance, " reduced" light vergence, "reduced" focal length, etc. is something that is basic and merits the study of every ophthalmologist. Unfortunately, the subject is hardly touched upon in the standard books on ophthalmology. It is true that for a lens in air, the reduced focal length is equal to the actual focal length and its significance and use may be ignored. But when studying the dioptrics of the cornea or of the eye as a whole where the reduced focal length is not equal to the actual focal length, a knowledge of this subject is essential. Books which do not point out the difference between the two systems create for the studentreader unnecessary difficulties and sometimes confusion.

For example, in Duke-Elder's Text-book of Ophthalmology, vol. I, page 748, there are tables contrasting the finding of Tscherning with those of Gullstrand relative to the position of the cardinal points, the dioptric powers of the components, etc. of the schematic eye. The table gives Tscherning's figure for the refractive power of the crystalline lens (in situ) as 16.01 D. and Gullstrand's as 19.11 D. This apparently means that Gullstrand using different data for his calculations found the power of the crystalline lens to be 3.10 dioptres more than what Tscherning found. But there is no mention of the fact that Tscherning used " actual " focal length for his power determination and Gullstrand used used "reduced" focal length. In 
fact, if the lens power as found by Tscherning be changed to "reduced" power it comes out even more than Gullstrand's, being $21.40 \mathrm{D}$. That is, the reduced power of the crystalline lens, using Tscherning's data for radii, indices and thickness is more by $2: 29 \mathrm{D}$. than the value found by Gullstrand.*

The method of calculating reduced surface power directly is very muctr simplified by what has been called the "dam" formula, $\mathrm{D}=\mathrm{aM}$. Here $\mathrm{D}$ stands for dioptres of power (reciprocal of "reduced" focal length) "a" stands for the difference between the two indices, (i.e. "a" amount 2 nd index is above 1st index) and $M$ stands for curvature in metrecs. The-latter is obtained by dividing the radius into 100 , or 1,000 or 40 depending upon whether the radius is given in $\mathrm{cm}$. in $\mathrm{mm}$. or in inches.

As an illustration we may apply this formula to finding the "reduced" power of the crystalline lens, using Tscherning's data which are as follows : $r 1=10.2 \mathrm{~mm}, \mathrm{r}_{2}=6.22 \mathrm{~mm} . \quad \mathrm{t}=4.06 \mathrm{~mm}$. $\mathrm{n}$ of lens $=1.42$, and $\mathrm{n}=$ of aqueous - vitreous equals 1.3365 .

For first surface $D=(1.42-\overline{1} \cdot 3365) \times \frac{1,000}{10.2}$; this works out to $8 \cdot 19$.

For second surface $D=(1.3365-1.42) \times \frac{1,000}{-6.22}$; this works out to $13 \cdot 42$.

The sum of the two surface powers is $21.61 \mathrm{D}$. The effect of thickness reduces the total power by 0.21 , giving a power of $21.40 \mathrm{D}$.

\title{
RESTORATION OF THE CHAMBER AFTER INTRA-CAPSULAR EXTRACTION
}

BY

\author{
Prof. A: Kettesy \\ DEBRECEN, HUNGARY
}

- IT is well-known, since doing intra-capsular cataract extraction that we see delayed restoration of the anterior chamber more frequently than before.

This complication in the after-treatment has generally no signifi-. cance at all, more properly, less significance than in the extracapsular extraction, when there is the possibility of capsular or cortical particles left in the section. Nor has it any significance after the intra-capsular extraction either, if a shallow chamber is present if for only a short time. The well-known and easily

* The illustration taken from Duke-Elder's work is not meant as a criticism, but just to point out this omission even in the most scholarly and best known book on the subject. 